(Aus der Frankfurter Poliklinik für Nervenkranke.)

\title{
Verschiedene Vulneral,ilität bzw. Giftaffinität der Nerven oder Gesetz der Lähmungstypen?
}

Von

Siegmund Auerhach (Frankfurt a. M.)ํ).

(Eingegangen am 1. Juni 1918.)

M. H.! Auf der letzten Jahresversammlung der Gesellschaft Deutscher Nervenärzte in Bonn (September 1917) sagte ich, daß ich den von O. Foerster zur Erklärung des zeitlich und graduell verschiedenen Rüickganges der Lähmungen in den einzelnen Muskelgruppen nach Schußverletzungen aufgestellten Begriff der verschiedenen ,Vulnerabilität" der Nervenfasern nicht akzeptieren könne. Tch gab diesem Bəgriffe das Epitheton ,mystisch" und hetonte, daß jene regelmäßige Erscheinung restlos durch das von mir für die gesamte Neuropathologie aufgestellte ,Gesetz der Lähmungstypen“" zu erklären sei. Dieses Gesetz lautet: „Diejenigen Muskeln bzw. Muskelgruppen erlahmen am raschesten und vollkommensten bzw. erholen sich am langsamsten und am wenigsten, die die geringste Kraft (ausgedrückt durch das Muskelgewicht) besitzen und ihre Arbeitsleistung unter ungünstigen physikalischen. physiologischen und anatomischen Bedingungen zu vollbringen haben, während die in dieser Beziehung besser gestellten Muskeln von der Lähmung größtenteils verschont bJeiben."

In seinem Schlußworte hielt Foerster (vgl. den Eigenbencht im Neurol. Centralbl. 1917, Nr. 20) den Begriff der verschiedenen Vulnerabilität der Nervenfasern für verschiedene Muskeln unbedingt aufrecht und hielt ferner aufrecht, daß die Fasern für die distalen Muskeln vulnerabler seien als die für die proximalen. Der Eigenbericht lautet dann weiter : ,Die Auffassung A u erbach s steht übrigens nicht im Widerspruch zu der Auffassung des Vortragenden, weil die distalen Muskeln dem Volumen nach weit schwächer sind als die proximalen, nur kannn natïrlich nicht, wie Auerbach es annimmt, das Muskelvolumen an

t) Vortrag gehalten auf der 43. Wanderversammlung der südwestdeutschen Neurologen und Psychiater in Baden-Baden am 26. V. 1918. 
sich maßgebend für die Vulnerabilität der Nervenfasern sein. Wohlgemerkt, gilt das vom Vortragenden Vorgetragene nur für traumatische Schädigungen, nicht aber für toxische und infektiöse; hier kommen noch ganz besondere Affinitäten in Betracht."

Herr Curschmann stimmte Foerster zu. Nach ihm (vgl. den erwähnten Bericht) entspricht diese Vulnerabilität ,der Kompressionsschädigungsmöglichkeit (z. T. bei professioneller Lähmung) einerseits und der verschiedenen Erkrankungsbereitschaft verschiedener Nerven auf toxische Einflüsse hin. C. verweist auf die größere Vulnerabilität des $\mathrm{N}$. peron. gegenüber dem $\mathrm{N}$. tibial. und des $\mathrm{N}$. axillaris gegenüber anderen Nerven des Plexus".

Tch vermute, daß weder Foerster noch Curschmann meine Publikationen $^{1}$ ) über diese, wie ich zugebe, dem Verständnisse nicht ganz leicht zugängliche Materie hinreichend bekannt waren, wie das auch bei Herrn Gerhardt der Fall war, als er vor 2 Jahren hier seinen Vortrag hielt , t'ber die Beeinflussung organischer Lähmungen durch funktionelle Verhältnisse" (Deutsche Zeitschr. f. Nervenheilk., 55, 226 und Sitzungsbericht der Physikal. med. Gesellschaft zu Würzburg vom 9. XI. 16). Nachdem ich Herrn Gerhardt auf meine Arbeiten, die ihm entgangen waren, aufmerksam gemacht hatte, gab er sofort $\mathrm{zu}$, daß meine Lähmungstheorie die von ihm aufgeworfenen Fragen der Motilität in plausibelster Weise zu erklären imstande sei. Ich möchte annehmen, daß es den Herren Foerster und Curschmann ebenso ergehen wird, wenn sie sich nur mit meinen ausführlichen Veröffentlichungen vertraut machen wollen.

M. H.! Es kann hier nicht meine Aufgabe sein, den ganzen Weg noch einmal zu durchwandern, der mich zur Aufstellung des Lähmungsgesetzes geführt hat. Jeder, der sich mit Lähmungen zu beschäftigen hat - und wer wäre das nicht in diesem Kreise - wird sich, wenn er den Erscheinungen nicht ratlos gegenüber stehenbleiben will, mit meinen Erörterungen befassen müssen. Ich glaube versichern zu dürfen, daß er dies nicht bereuen wird. Hier werde ich mich so kurz fassen, als es das Vortragsthema gerade noch zuläßt

Was bedeuten denn Ausdrücke wie verschiedene „Vulnerabilität“, „Giftaffinität" und ,Erkrankungsbereitschaft" der Nerven? Das sind doch nur Umschreibungen des Tatbestandes, der Erscheinungen, aber keine Erklärungen. Ich finde es wohl begreiflich, daß ein Hautnerv

1) Die Hauptursachen der häufigsten Lähmungstypen. Volk manns Samm. lung klin. Vorträge Nr. 633/634, 1911. - Zur Pathogenese der postdiphtherischen Ak kommodationslähmung. Deutsche Zeitschr. f. Nervenheilkunde 49, 94. - Die Aufbrauchtheorie und das Gesetz der Lähmungstypen 53, 449. - Zur Lehre von den Lähmungstypen 57, 101. - Warum beobachtet man Lähmungen des $\mathrm{N}$. peron. viel häufiger als solche des N. tibialis? Deutsche med. Wochenschr. 1916, S. 1228. 
oder auch ein oberflächlich liegender gemischter Nerv wie der N. radialis an seiner Umschlagstelle am Oberarm oder der N. ulnaris am Epicondylus humeri eher verletzt werden kann als ein tiefliegender Nerv, da $B$ er ,vulnerabler" ist als der letztere. Die Annahme einer verschiedenen „Vulnerabilität" bringt mich aber doch in meinem kausalen Denken keinen Schritt vorwärts, wenn ich mir die jetzt in zahlreichen Fällen ganz regelmäßige Beobachtung erklären soll, daß nach Abschuß des $\mathrm{N}$. ischiadicus am Oberschenkel oder nach Resektion eines seinen ganzen Querschnitt durchsetzenden Callus und darauffolgender Naht dieses Nerven stets, falls Regeneration eintritt, zuerst die vom N. tibialis innervierten Plantarflexoren des Fußes ihre Funktion wiedererlangen, und erst viel später, wenn überhaupt, die vom $\mathrm{N}$. peron. beherrschten Dorsalflexoren und Abductoren. Diese béi Läsion des Gesamtquerschnittes des $\mathrm{N}$. ischiad. - bei Verletzungen einzelner Bahnen kommen natürlich rein topographische Gesichtspunkte in Betracht regelmäßige Erscheinung ist doch unmöglich damit zu erklären, daß man annimmt, die Bahn des N. peron. im Ischiadieus sei ,vulnerabler" als die des $N$. tibial. Sie ist auch nicht zu erklären mit der größeren Distanz, welche die auswachsenden Nervenfasern zu durchlaufen haben, ebensowenig mit der größeren Entfernung vom trophischen Zentrum der zugehörigen Vorderhornganglienzellen. Denn es wird doch niemand behaupten wollen, daB in dieser Beziehung Unterschiede zwischen dem N. tibial. und dem N. peron. bestehen. Auch die bessere Gefäßversorgung der Tibialisgruppe gegenüber der Peroneusgruppe kann nicht befriedigen, da nach der Ro nx schen Lehre von den Erhaltungs- und Gestaltungsfunktionen die voluminöseren Muskeln eo ipso stärkere und zahlreichere Gefäße erhalten, als die weniger umfangreichen; hierin kann aber keine Minderwertigkeit der letzteren erblickt werden.

Dem Verständnisse zugänglich wird die erwähnte Beobachtung erst durch folgende Uberlegungen: Die Kraft der Plantarflexoren, ausgedrückt durch das Muskelvolumen bzw. das Muskeltrockengewicht ist nach den Bestiminungen der (cebrüder $W$ eber ${ }^{1}$ ) größer als die Kraft aller übrigen Unterschenkelmuskeln zusammengenommen $(733: 537)$. Nach Frohse und M. Fränkel'2) verhält sich das Gewicht der Plantarflexoren des Fußes (Gastroenemius + Soleus + Plantaris) zu dem der Dorsalflexoren (Tibial. ant. + Ext. digit. long. + peron. tertins) wie $795: 196$, also wie $4: 1$. Die $\mathrm{Mm}$. peron. long. et brevis (Abductoren bzw. Pronatoren) gehören zu den schwächsten; ihre Gewichtszahl beträgt nach Frohse und Fränkel 123, nach den Gebrüdem Weber 137,2. Berücksichtigt man nun außerdem, daß die Wadenmuskeln mit der Schwere

1) Mechanik der menschlichen Gehwerkzenge. Göttingen 1836.

2) Die Muskeln des mensehlichen Beins. Handbuch d. Anat. v. Bardeleben 2, II. Abt., II. Teil B., Jena 1913. 
arbeiten, die ohnehin schwachen Peronei aber gegen diese und als $\mathrm{Ab}$ ductoren den Fuß von der Medianebene des Körpers zu entfernen haben. welche Bewegung natürlich eine größere Arbeitsleistung darstellt als die Bewegung nach der Medianebene des Körpers hin, so wird man es begreiflich finden, daß ceteris paribus die Plantarflexoren des Fußes ihre Funktion früher wiederaufnehmen als die Dorsalflexoren. Das Erfolgsorgan des N. tibialis ist eben viel kräftiger und hat außerdem seine Arbeit unter günstigeren Bedingungen zul leisten als dasjenige des $\mathrm{N}$. peroneus.

Ein weiteres Beispiel, das uns bei den Massenbeobachtungen dieses Krieges jetzt tagtäglich vor Augen tritt: Bei Abschüssen des N. radialis am Oberarm bzw. bei einem seinen ganzen Querschnitt durchsetzenden Nervencallus, Resektion desselben und darauffolgender Naht treten regelmäßig zuerst die Strecker des Carpus wieder in Tätigkeit, viel später die Strecker der Finger. Weshalb? Weil die Kraft der ersteren, ausgedrückt durch ihr Muskelgewicht, sich zu der der letzteren verhält wie 114:50 [Fränkel und Frohse $\mathrm{e}^{1}$ ]]. Die Fingerstrecker sind die schwächsten sämtlicher langen Fingermuskeln. Hierzu kommt noch, daß die Kraft, welche sie aufzuwenden haben, um ihre Arbeit gegen die Schwere zu vollbringen, eine relativ größere ist, als die von den Handstreckern zu leistende, weil ${ }^{2}$ ) in der natürlichen Ruhelage die Grundphalangen der Finger sich in leichter Beugestellung befinden, während die Handstrecker in dor Ruhelage in Folge ihrer Uberlegenheit an Masse äber die Handbeuger bereits eine geringe Dorsalflexion einnehmen.

Es handelt sich also bei meiner Auffassung der Dinge gar nicht um einen Vergleich zwischen distalen und proximalen Muskeln, wie Foerster meint (vgl. oben!), sondern um einen Vergleich der distalen Muskeln unter sich, auch ist natürlich das Muskelvolumen an sich für mich keineswegs allein maßgebend für die sog. Vulnerabilität der Nerven. fasern. Mir kam es darauf an, die Momente zu ermitteln, die es bedingen, daß nach völliger Leitungsunterbrechung im innervierenden Nervenstamm zwei von ihm versorgte Muskelgruppen der distalen Extremitätenabschnitte ihre Finktion zu ganz verschiedenen Zeiten und in ganz. verschiedenem Grade wiedererlangen; ich behaupte sogar, daß sie sich so verhaltęn müssen. In einer größeren Arbeit aus neuerer Zeit über „,Die Kriegsverletzungen der peripheren Nerven", die aus der Wiener Nervenheilanstalt Maria-Theresien-Sehlössel stammt und auf diese Fragen eingeht, hebt Wexberg ${ }^{3}$ ) die Berechtigung meiner Theorie hervor.

1) Die Muskeln des menschlichen Arms. Handbuch d. Anat. v. Bardeleben z, II. Abt., II. Teil, Jena 1913.

2) Volkmannsches Heft l. c. S. 154 u.155.

3) Zeitschr, f. d. ges. Neurol, u. Psych. 36, 345. 1917. 
Für die traumatische Gruppe unter den peripheren Lähmungen kommt das Gesetz der Lähmungstypen, wie ich bereits angedeutet habe, nur in beschränktem Umfange in Betracht. Durch eine Verletzung kann jeder Nerv und jeder von ihm abhängige Muskel gelähmt werden, ganz ohne Rücksicht auf seine spezielle Muskelkraft und die Arbeitsbedingungen, unter denen er sich zu betätigen pflegt. Es kann sich nur um solche Fälle handeln, in denen das Trauma ei ne n mehrere Muskeln innervierenden Nerven nachweislich in seinem ganzen Querschnitte lädiert oder einen Nervenplexus in toto getroffen hat, oder wenn bei partieller Verletzung eines solchen durch einen autoptischen Operationsbefund konstatiert werden kann, welche Äste verschont geblieben sind. Allgemeine Gülltigkeit hat das Lähmungsgesetz jedoch für die übrigen typischen Lähmungen der peripheren Nerven, insbesondere für die durch eine Polyneuritis bedingten. Und hiermit komme ich auf den Begriff der „Giftaffinität“ und der ,Erkrankungsbereitschaft" zu sprechen. Auch hier fasse ich mich so kurz wie möglich und verweise auf meine einschlägigen Arbeiten.

Wir nehmen an, daß die verschiedenen Gifte, mögen sie toxischer oder infektiöser Natur sein, eine verschieden große Affinität zu einzelnen Organen oder Organsystemen haben oder umgekehrt. So gibt es Gefäß-. Blut-, Muskelgifte usw, selbstverständlich auch Nervengifte, und unter diesen wieder solche, die sich mit Vorliebe in der Gehirn- oder Rückenmarksubstanz verankern, andere, welche die peripheren Nerven bevorzugen. Warum das eine Gift mit Vorliebe oder ausschließlich dieses oder jenes Organ befällt, wissen wir, von einigen Ausnahmen abgesehen, nicht und nehmen deshalb zu dem Begriffe der differenten Giftaffinität unsere Zuflucht. Unser Kausalbedürfnis kann und muß sich hiermit vorläufig zufrieden geben. Es kann ihm aber unmöglich zugemutet werden, anzunehmen, daß ein und dasselbe Gift eine besondere Verwandtschaft zu bestimmten peripheren Nerven oder Nervenästen eines Extremitätenabschnittes besitzt, daß es aber andere derselben Gliedmaße, die anatomisch und chemisch genau ebenso konstruiert sind, verschont. Besonders bemerkenswert ist, daß, wenn bei der gewöhnlichen Polyneuritis ein vom N. peron. profund. innervierter MuskeI intakt bleibt, es der relativ kräftigste $M$. tibial. anticus ist, obgleich auch er die Anziehungskraft der Erde zu überwinden hat. Das kann man doch wirklich nicht mit einer verschiedenen Giftaffinität oder Erkrankungsbereitschaft der Nervenfasern erklären. Es wäre doch mehr als gezwungen, anzunehmen, daß die die Mm. extens. digit. long. et brevis und extens. halluc. long. et brevis versorgenden Nervenäste des $\mathrm{N}$. peron. profund. eine größere Affinität zu dem betreffenden Gifte haben als die in den $M$. tibial. anticus eindringenden, daß das krankmachende Agens jene befällt und vor diesen haltmacht! Das Muskelgewicht des 
Tibial. anticus verhält sich aber zu dem der Zehenstrecker wie 122 :84:38 Ebensowenig kann man die von $O p$ penheim in seinem Lehrbuche (6. Aufl., S. 672) noch als ,überraschend" bezeichnete Tatsache mit einer verschiedenen Giftaffinität der betreffenden Nervenfasern erklären, da $\beta$ bei der polyneuritischen Radialislähmung von den verschiedenen, unter der Herrschaft dieses Nerven stehenden Muskeln ein Teil gelähmt sein kann, während die anderen ihre Bewegungsfähigkeit bewahren, daß diese Lähmung sich sogar im Beginne auf den Ext. digit. commun. beschränken kann. Nach dem vorhin Gesagten (vgl, oben) ist diese Tatsache keineswegs überraschend, sie muß vielmehr als ein notwendiges Postulat erscheinen, wenn die von mir aufgestellte Lehre richtig ist. Mit ihr gelingt es auch, zwanglos den Lähmungstypus bei der Bleilähmung und vielen anderen Lähmungsformen dem Verständnisse zugänglich zu machen. Die von Edinger geltend gemachte quantitative Funktionssteigerung, der „Aufbrauch", ist hierzu durchaus entbehrlich und überdies auch öfters nicht zutreffend. Es genügen völlig die von mir aufgedeckten ,,inneren Gründe", die ,,generellen Faktoren", deren Existenz Gerhardt (l. c.) vermutet hatte, die Qualität der Einzelleistungen. Wie wenig berechtigt die Annahme einer verschiedenen Giftaffinität oder Erkrankungsbereitschaft der Nervenfasern ist, zeigt auch die von vielen Beobachtern gemachte Feststellung, daß bei der Polyneuritis anscheinend funktionstüchtige Muskeln bei genauer Untersuchung sich oft auch als leicht geschwächt erweisen und eine deutlich herabgesetzte elektrische Erregbarkeit zeigen. Zum völligen Versagen aber kommt es nur bei den weniger kräftigen und unter ungünstigen Umständen arbeitenden Muskeln.

Nach dem Ergebnis meiner Untersuchungen, die ich hier natürlich nur in nuce vortragen konnte, sollte man Begriffe wie verschiedene „Vulnerabilität" oder „Giftaffinität lassen. Sie sind für unser kausales Denken entbehrlich geworden, nachdem es gelungen ist, sie durch exakte physikalische und physiologisch-anatomische Vorstellungen zu ersetzen. Daß dies einen Fortschritt in der Erkenntnis bedeutet, wird wohl niemand bestreiten können. 